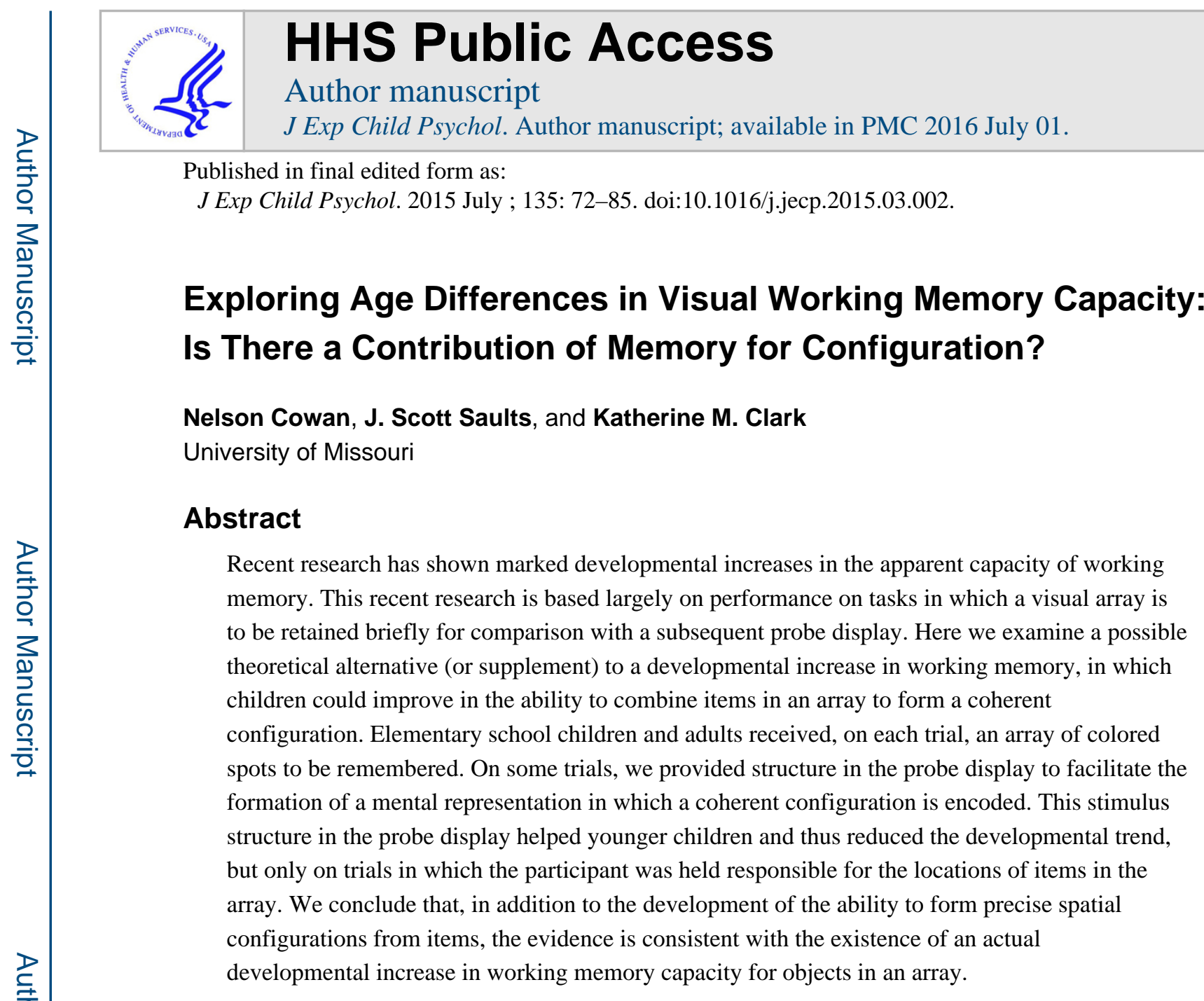

\title{
Keywords
}

working memory; chunking; grouping; visual short-term memory

The present work focuses on the developmental role of one potential factor that might influence working memory ability for a visual array of simple objects: the ability to combine multiple items in a display to form an overall mental configuration. This configuration would be one form of grouping items to form a new, larger unit in memory, reducing the load on working memory similar to what one can do by chunking together items based on prior knowledge (Miller, 1956).

Children from the late preschool years through adulthood can carry out some of the same sorts of tasks designed to examine working memory, which is essentially the small amount of information that can be held in mind at once. Clearly, working memory ability increases

(C) 2015 Published by Elsevier Inc.

Correspondence: Nelson Cowan Department of Psychological Sciences University of Missouri McAlester Hall Columbia, MO 65211 Tel. 573-823-4913 CowanN@ missouri.edu.

Publisher's Disclaimer: This is a PDF file of an unedited manuscript that has been accepted for publication. As a service to our customers we are providing this early version of the manuscript. The manuscript will undergo copyediting, typesetting, and review of the resulting proof before it is published in its final citable form. Please note that during the production process errors may be discovered which could affect the content, and all legal disclaimers that apply to the journal pertain. 
steadily during this age range (e.g., Gathercole, Pickering, Ambridge, \& Wearing, 2004). Working memory is of special importance because its capacity limits are thought to constrain the kinds of concepts that an individual can understand, or problems that he or she can solve (e.g., Halford, Cowan, \& Andrews, 2007). If we understood the reasons for working memory capacity development, that understanding could in turn shed light on the nature of capacity limits in adults, the mechanisms of cognition in children, and the best course for improving educational practices (e.g., Cowan, 2014).

There is a growing body of evidence on the development of working memory in changedetection tasks (after Luck \& Vogel, 1997), in which a briefly-presented array of objects to be remembered is followed by a probe to be judged present or absent from the array (Cowan et al., 2005; Cowan, Fristoe, Elliott, Brunner, \& Saults, 2006; Cowan, Morey, AuBuchon, Zwilling, \& Gilchrist, 2010; Riggs, McTaggart, Simpson, \& Freeman, 2006; cf. Shore, Burack, Miller, Joseph, \& Enns, 2006). Items in an array, however, sometimes can be combined to form a configuration, at least by adults (e.g., Brady \& Tenenbaum, 2013; Chong \& Treisman, 2003, 2005; Jiang, Olsen, \& Chun, 2000; Woodman, Vecera, \& Luck, 2003; Xu \& Chun, 2007). If older participants use a multi-item configuration more readily than young children do, then we might expect stimulus cues that encourage a configuration to help children use them, thereby reducing age differences in working memory.

Alternatively, if no age groups are ordinarily using a configuration in a particular situation, then cues encouraging a configuration might be used only by more mature participants, thereby increasing age effects in working memory.

There is some evidence suggesting that it is possible to dissociate effects of configuration from other aspects of working memory load. Jiang, Capistrano, and Palm (2014) recently found that array configuration knowledge did not differ between children with and without autistic spectrum disorders, even though capacity was impaired in those with autistic spectrum disorders. We sought to use a similar research strategy, applied to a paradigm that we used previously (Cowan et al., 2010), to determine whether cues to the configuration would similarly help participants of all ages or would help one age group more than another. The latter outcome would indicate a developmental change in the use of configuration to assist in memory span. In principle, it would even be possible that age differences in span might come largely or entirely from age differences in the ability to use knowledge of the configuration.

\section{Present Experiment}

Consider a stimulus setup like the one that we used in the present study (modified from Cowan et al., 2010 to study context effects; see Figure 1). Each trial included an array to be studied, containing 4 differently-colored objects: in particular, 2 circles and 2 triangles. This array was followed by a probe display that included a single colored object, or probe item. The probe item always matched one array item in both location and shape, but the nature of the color of the probe varied depending on the condition. Judgments were to be made about where, if anywhere, the probe item appeared in the prior array. The answer was to be given with a mouse click. 
An individual might notice things about the configuration of stimuli in the initial array. On some trials, for example, the individual might notice that the circles both have light colors (red, yellow) whereas the triangles both have dark colors (blue, green). Alternatively, the individual might encode the overall configuration formed by the constellation of colors, and then use a memory of this configuration to reproduce the color needed to compare with the probe. Any such knowledge about the configuration could be useful when the memory test is given.

The main innovation of the study is the provision on some trials of cues to the configuration of the array (as shown in Figure 1). The task was basically to identify the location of an identical object in the array, or indicate its absence from the array. In a no-context condition, there was only one item in the probe display, the probe item. In a context condition,

however, an additional set of cues was offered: the shapes presented at all other locations in the array, albeit with the color information missing. The issue we examine is the benefit of this additional set of contextual cues to the configuration of objects, and the developmental change in the use of these additional cues.

The experiment was designed to provide a distinction between memory for features and memory for integrated objects with features bound together (cf. Cowan et al., 2013; Luck \& Vogel, 1997; Wheeler \& Treisman, 2002). It would be possible for individuals to form a configuration of colors either with or without knowing the corresponding shapes. One of the conditions of the experiment (other-shape's-color, to be explained shortly) allowed errors indicating that the shape was not known, and the context condition provided assistance by supplying the potentially forgotten shape information.

Using just two shapes allowed us to ask about the role of attention in forming a configuration. On $80 \%$ of the trials, the probe item was a colored circle, presented where a colored circle had been in the array and on the other $20 \%$ it was a colored triangle presented where a colored triangle had been in the array. Cowan et al. (2010) found that participants from the elementary years up focused more attention on the more-often-tested shape and remembered more of the items of that shape. By offering the shape information at the time of testing in the context condition, we can determine whether any configuration that is formed in working memory includes all items, or only items of the more-often-tested shape. If the latter is the case, one could expect the context to be more important to supplement and support working memory information when the probe is presented in the less-often-tested shape.

We included four kinds of test probes (which were common to both no-context and context conditions) and there is reason to expect that the use of contextual information might differ between these probe types, as follows.

\section{Changed-location probes}

In one type of test probe, the color of the probed item has changed to the color of the other array item of the same shape from the array. For this probe type, context can help in several ways. First, without context the participant might know that the color was not in the same location as the probe, but might not recall exactly where that probed color actually occurred 
in the array. The context objects restrict the possible responses, which should improve accuracy. Beyond that, the context objects may serve as cues reminding the participant that the probed color was present in the array, just not in the probed location. Potentially, moreover, that use of the context theoretically could be superior in the presence of a moreoften-presented probe shape (circle) compared to a less-often-presented probe shape (triangle), if participants tend not to form configuration information for the less-often-tested shape. With development, there theoretically might be a decrease in the need for external contextual cues, for all items or just for one shape.

\section{Other-shape's-color probes}

In a second type of test probe, the color of the probed item has changed to the color of an array item of the opposite shape. If the participant remembers the configuration of colors but forgets the associated shapes, it could lead to an incorrect response of clicking on the location where the color resided in the array, even though that object was the wrong shape; the correct answer is that the probe object was not anywhere in the array. The contextual cue can prevent that kind of error by showing the shape in the array at each location.

The use of context may be more difficult for other-shape's-color probe trials than for the changed-location trials. In the changed-location trials, the participant can use the context to supply precise location information and combine it with information in working memory to determine whether there is a match for the probe. If a color match is found, the participant will be correct to choose the location of the match, even without considering shape. If shape is considered as well, an exact match will be observed. In contrast, the other-shape's-color condition involves contradictory information: the colors match at a location at which the shapes do not match. The matching color and mismatching shape imply that the probe object was not in the array, but even adult participants do not always correctly use this process of elimination; when they do not find a match, they sometimes seem to resort instead to random guessing (Chen \& Cowan, 2013). It is quite possible that this kind of defective inference occurs more frequently in children than in adults, given incomplete maturation of frontal lobe areas of the brain (e.g., Casey, Giedd, \& Thomas, 2000).

\section{New-color probes}

In a third probe type, the probe is presented in a color that was not anywhere in the array, and the correct answer is to indicate as much. To answer this problem correctly, information about the location of the colors in the array is not needed. If context helps in these trials, that can only occur because the context reminds the participant of an overall configuration in working memory, serving as a reminder for more of the colors that were presented. This context could help rule out the new color by process of elimination. Although this type of cueing seems usual in long-term memory, however, we know of no evidence indicating whether it should occur in working memory or not. It might not occur in our study in particular if the potential function of a context cue is to revive an item from long-term to working memory; there may be no sufficiently strong long-term memory representation of the current trial in our study to draw upon, given the repeated presentations of the same set of objects in different arrangements on each trial. 


\section{No-change probes}

In a fourth probe trial type, the probe matches the item that appeared at the same location in the array. The correct answer is to click on the probe location. Context conceivably could help to remind the participant of the color of objects elsewhere in the array, which could help to rule out these other locations as the location of the probe item. Again, we know of no evidence indicating whether this kind of cueing should occur in working memory at any age.

\section{Additional design considerations}

These four probe types, each occurring for objects of the more-often-probed and the lessoften-probed shape, should reveal a great deal about what configurational information might be used, and how that use changes with childhood development. Several additional factors helped guide our specific design. Our decision to make color primary and shape secondary in the design stemmed in part from a previous finding in adults suggesting that a spatial configuration of colors can be formed more easily than a spatial configuration of shapes (Cowan, Saults, \& Blume, 2014). Another reason to make color primary is that we wished to make our stimulus conditions resemble those of Cowan et al. (2010) to allow comparisons of benchmark findings across studies. Apart from contextual cueing, our present changedetection method was identical to that of the $80 \%$ relevant, $20 \%$ irrelevant test probe blocks of Cowan et al. (2010). Cowan et al. found that children as young as 7 years of age were able to concentrate on the relevant shape at the expense of the less-relevant shape as well as adults could. Yet, these young children recalled far fewer items from the array. These benchmarks were replicated here.

Finally, we avoided providing non-probed colors along with the shapes as cues, given that this more complete contextual information actually overwhelms participants with interference, reducing performance overall rather than assisting it (Wheeler \& Treisman, 2002).

\section{Method}

After each array, a probe item was presented at the location of a prior array item of the same shape. The task was to make a mouse click that was rich in information, indicating where to find the array item matching the probe in both color and shape. It could belong in the location in which the probe item was presented, as in the Figure 1 example; in a different array location out of 12 possible locations; or nowhere in the array, in which case the participant was to click on the door icon (based on a cover story to make the task interesting for children, described below). The most important change in the present experiment was that, in either the first or the second half of the session, the probe presented at the end of each trial was accompanied by markers indicating the shapes of the items that had appeared in the array at the other specific locations, in a black outline form that did not indicate the color of the item that had appeared. These contextual cues restricted the possible response locations from 12 to 4 , and provided information about the shape at each location. 


\section{Participants}

The final sample included 104 participants with $\mathrm{n}=26$ in each of four age groups: Grades 1-2 (age $\mathrm{M}=7.72$ years, $\mathrm{SD}=0.76 ; 17$ female, 9 male), Grades 3-4 (age $\mathrm{M}=9.28, \mathrm{SD}=0.78 ; 14$ female, 12 male), Grades 5-7 (age $\mathrm{M}=11.63$ years, $\mathrm{SD}=0.93 ; 15$ female, 11 male), and adults from the community (age $\mathrm{M}=37.97$ years, $\mathrm{SD}=6.24 ; 22$ female, 4 male). A few additional children chose not to complete the experiment, including 2 in Grades 1-2, 3 in Grades 3-4, and 1 in Grades 5-7. An additional 4 children were lost from the sample because of equipment malfunction (3 in Grades 3-4 and 1 in Grades 5-7).

\section{Apparatus, Stimuli, and Procedure}

The instructions indicated that the participant would usually be tested on circles and should attend to that shape and ignore the triangles. As in Cowan et al. (2010), each trial began with the presentation of a small, hollow circle for $1000 \mathrm{~ms}$, which served as a fixation point and a reminder of the shape to be attended. Next came a 500-ms display of an array to be remembered, with four differently-colored shapes (two solid circles and two solid triangles) presented centered within $1.77-\mathrm{cm}$-wide squares that were found at random locations within a regular grid with 3 rows of 4 squares each, arranged evenly within an area on the screen $8.19 \mathrm{~cm}$ wide and $6.72 \mathrm{~cm}$ tall. Each colored circle and triangle (equilateral with point down) was sized to fit within an imaginary $0.57-\mathrm{cm}$-wide box. The colors of the objects were selected on each trial without replacement from the set, black, white, red, blue, yellow, green, orange, purple, brown and pink. This array was followed by a 1500-ms blank screen with the same shade of gray that was used as a background throughout the trial, and then by the probe display until a response was made.

The experiment was presented as a game in which the grid of squares represented desks in the classroom, with each colored shape representing a child at a desk in the classroom. When the probe display was presented, the task was to determine whether the one particular "object-child" (colored shape) shown in the probe display belonged at the desk shown, at another desk, or nowhere in the classroom, according to the most recently studied array. The response was made by clicking on the desk at which the object-child belonged. In order to allow a response indicating that the object-child belonged nowhere in the classroom, a 1.38$\mathrm{cm}$-wide $\times 2.35-\mathrm{cm}$-tall door icon appeared to the right of the grid in the probe display; clicking on the door indicated that the probe object-child was to be sent to the principal to be assigned to some other classroom. The probe display remained on until a response was given by mouse click on one of the squares or on the door.

Trials differed by the nature of the probe object. On $80 \%$ of the trials it was a colored circle, presented where a colored circle had been in the array, and on $20 \%$ of the trials it was a triangle, presented where a colored triangle had been in the array. Within these attendedand ignored-shape conditions, there was the same distribution of probe conditions: $20 \%$ probes that were identical to a probe of the same shape that had appeared at the probe's location, but in a color that was used for the other item of that same shape (location-change probes); $20 \%$ probes with the color of an item that had been used for an item in the array of the other shape (other-shape's-color probes); $20 \%$ probes that were in a color not found in the array (new-color probes); and $40 \%$ probes with no change from the array item that had 
appeared in that location (no-change probes). The correct response was to click on the location or "desk" in which the probe was presented on no-change trials, on the desk at which the probe object actually had occurred on location-change trials, and on the door icon on new-color or other-shape's-color trials.

The difference between no-context trial blocks, matching the procedure of Cowan et al. (2010), and shape-context trial blocks is that, in the latter case, the array items that had appeared at locations other than the one that contained the probe were noted by empty shape outlines that indicated the shapes, but not the colors, of the items that had appeared at those locations in the array. This context did not give away the correct answer, but it theoretically might provide cues to the memory of the array as a whole.

Each participant received a total of 50 practice and 300 test trials, divided into two trial blocks of equal length. Half of the participants in each age group received the no-context block before the context block, and the other half received the reverse order. Each trial block began with 25 practice trials, including a full complement of trials (e.g., 20 trials in which memory of a colored circle was tested and 5 trials in which memory of a colored triangle was tested). Accuracy feedback provided in practice trials was omitted from test trials. The test trials within each trial block were distributed in the same proportions as the practice trials and thus, for the attended (and ignored) shape probes in a trial block there were 48 (and 12) no-change, 24 (and 6) new-color, 24 (and 6) location-change, and 24 (and 6) othershape's-color test probe trials. Within each trial block, each consecutive run of 25 trials included one trial of each type, in random order.

\section{Results}

We present as our main analysis the proportion correct for each age group and condition. Next, we examine scores providing estimates of the number of items in working memory. Finally, we examine the pattern of errors in different conditions to gain more insight into the effects of context.

\section{Proportion Correct}

Proportion correct scores for each participant in each of the four age groups were calculated separately for no-context and shape-context trial blocks. Within each trial block, the proportion correct was calculated separately each of four types of trial differing in the type of probe: changed-location, other-shape's-color, new-color, and no-change trials. These proportions were entered into an ANOVA with a 4 (age group) $\times 2$ (context) $\times 4$ (probe type) design. The pattern across conditions was comparable to prior work (Cowan et al., 2010) and so for simplicity, ANOVA results will be limited to those that include age group as a factor.

The main effect of age group was significant, $\mathrm{F}(3,100)=17.16$, MSE $=0.22, \mathrm{p}<.001, \eta_{\mathrm{p}}{ }^{2}=.34$. Proportions correct for the four age groups increasing in age were $.48, .55, .62$, and .70 (pooled SEM=.02). Most importantly, however, there was a three-way interaction between the age group, the context, and the probe type, $\mathrm{F}(9,300)=2.95$, MSE $=0.03, \mathrm{p}<.005, \eta_{\mathrm{p}}{ }^{2}=.08$. 
The results corresponding to this interaction can be seen in Figure 2, with a different probe type represented in each of 4 panels of the figure, as marked.

To help interpret this interaction, the Age $\times$ Context interaction was examined separately for each probe type. There was no interaction for new-color probes, $\mathrm{F}(3,100)=2.13$, MSE $=0.03$, $\mathrm{p}>.1, \eta_{\mathrm{p}}{ }^{2}=.06$, or for no-change probes, $\mathrm{F}(3,100)=1.60, \mathrm{MSE}=0.03, \mathrm{p}>.1, \eta_{\mathrm{p}}{ }^{2}=.05$. However, the interaction was significant for the location-change probe type, $\mathrm{F}(3,100)=3.86$, $\mathrm{MSE}=0.04, \mathrm{p}<.05, \eta_{\mathrm{p}}{ }^{2}=.10$, and the other-shape's-color probe type, $\mathrm{F}(3,100)=3.40$, $\mathrm{MSE}=0.04, \mathrm{p}<.05, \eta_{\mathrm{p}}{ }^{2}=.09$. Figure 2 shows that the basis for this interaction differed for these two conditions. In the location-change trials, it was the younger age groups that benefitted more from the contextual markers of the shapes of unprobed array items. (Newman-Keuls post-hoc tests showed the context effect to be significant in the youngest group and the second-youngest group, $\mathrm{p}<.01$ in both cases, but it did not approach significance in the older two groups.) In the other-shape's-color probe situation it appears that only the oldest age group was able to make use of contextual markers. (Newman-Keuls tests showed the context effect to be significant only in the adults, $\mathrm{p}<.05$, and it did not approach significance in any child group.)

There was one other effect that included age as a factor in the main analysis, which was its interaction with the attention condition, $\mathrm{F}(3,100)=5.44, \mathrm{MSE}=0.05, \mathrm{p}<.005, \eta_{\mathrm{p}}{ }^{2}=.14$, unlike Cowan et al. (2010) who found no interaction in a condition identical to the present nocontext condition. Separate analysis of the no-context condition of the present data confirmed that the interaction was still present, $\mathrm{F}(3,100)=5.05$, $\mathrm{p}<.005$, MSE $=0.03, \eta_{\mathrm{p}}{ }^{2}=.13$. For both contexts, the pattern of results indicated that older participants benefitted more from attention to the probed shape than did younger participants (for Grades 1-2, attended $\mathrm{M}=.50$ vs. ignored $\mathrm{M}=.46$; for Grades $3-4, .57$ vs. .52; for Grades 5-7, .67 vs. .56; and for adults, .78 vs. .63; pooled SEM for attended and ignored $=.03$ and .02 , respectively).

Newman-Keuls tests showed the effect of attention to be significant only in the older two age groups, $\mathrm{p}<.001$ in each case. Attention and age did not contribute to any higher-order interaction. Our further analyses of items in working memory, however (below), suggest that this effect of attention is a scaling artefact given the different levels of performance in the task at the different age groups.

\section{Error Analysis}

An analysis of errors was carried out to help interpret the cases in which certain age groups benefitted from context according to the proportion correct. Table 1 shows the breakdown of erroneous responses in Groups 1 and 2, the youngest age groups, for the location-change probe trials. It is clear from the table that most of the benefit of context was to reduce the proportion of trials in which the participant correctly indicated that the probe belonged at a different location in the array, but was incorrect about which other location to select. There was relatively little change in the other error types when context was added (i.e., errors in which the child indicated no change or indicated that the probe was not found in the array). There were significant effects of the context, $\mathrm{F}(1,50)=35.92, \mathrm{MSE}=0.01, \mathrm{p}<.001 \eta_{\mathrm{p}}{ }^{2}=.42$, and of the type of error, $\mathrm{F}(2,100)=39.46, \mathrm{MSE}=0.09, \mathrm{p}<.001, \eta_{\mathrm{p}}{ }^{2}=.44$, and a marginal interaction between them, $\mathrm{F}(2,100)=2.80$, $\mathrm{MSE}=0.03, \mathrm{p}=.07, \eta_{\mathrm{p}}{ }^{2}=.05$. Not shown in the 
table, most of the wrong-location errors in the location-change, no-context condition were clicks on locations that did not have an object at all in the initial array. In the youngest two age groups these accounted for .13 and .08 of the responses; with context provided, they were reduced to .02 and .004 of the responses.

For the adults in the other-shape's-color condition, errors in which the participant incorrectly said that there was no change differed only a little between context conditions (no context, $\mathrm{M}=.13, \mathrm{SEM}=.02$; context, $\mathrm{M}=.11, \mathrm{SEM}=.02$ ). In contrast, errors in which the participant incorrectly said that the probe belonged elsewhere in the array were observably diminished on the basis of context (no context, $\mathrm{M}=.27, \mathrm{SEM}=.04$; context, $\mathrm{M}=.18$, $\mathrm{SEM}=.04$ ). There were significant effects of the context, $\mathrm{F}(1,25)=8.54$, MSE $=0.02, \mathrm{p}<.01, \eta_{\mathrm{p}}{ }^{2}=.25$, and of the type of error, $\mathrm{F}(1,25)=9.13$, MSE $=0.07, \mathrm{p}<.01, \eta_{\mathrm{p}}{ }^{2}=.27$, but they did not significantly interact, $\mathrm{F}(1,25)=2.69$, MSE $=0.03, \mathrm{p}=.11, \eta_{\mathrm{p}}{ }^{2}=.10$.

For adults, in a fairly high portion of the aforementioned other-array-location errors, the participant clicked on the location in which there had been an object with the same color as the probe but a different shape (no context, 0.17 ; context, 0.08 ). This last, 0.08 figure can be considered to reflect careless errors because working memory is not needed to prevent this type of mistake; just vigilance and maintenance of the task goal are needed to realize that a probe cannot belong to a location with the opposite shape in it. One might worry that children would often make this "careless" error and, for that reason, not make use of the context cue for the other-shape's-color condition. The children were not more careless than adults in this regard: the "careless" error in the presence of context was made in the four age groups on $0.05,0.06,0.04$, and .08 of the trials, respectively. Instead of careless errors, the younger children simply made more random errors than did the older children and adults in this condition.

In understanding why there was no effect of context for young children in the other-shape'scolor condition, note that the correct answer was that the probe was not in the array, so that there was no issue regarding the spatial precision of representations in the array. In the location-change condition, in contrast, the context could sharpen the spatial knowledge about where the probe had appeared in the array.

In sum, the main function of the context was to diminish two kinds of location-related errors at different ends of our age range, apparently for different reasons.

\section{Items in Working Memory}

Cowan (2001) described a simple measure that can be used to estimate the number of items in working memory in tasks like the present one, termed $k$. Assume that when something changed from the array to the probe, that change was detected with probability $\mathrm{p}$ (hits). Further assume that when nothing changed, that change was incorrectly indicated with probability p(false alarms). The assumption is that when the item from the array that is probed is in working memory, the participant will know whether that item has changed or not, and otherwise the participant will guess at a certain fixed rate. For an array with $S$ items, these assumptions yield $k=S[\mathrm{p}$ (hits)-p(false alarms)], which is called a double high-threshold model (Rouder et al., 2011). To estimate $k$, as in Cowan et al. (2010), it was necessary to 
combine information from color-change, location-change, and other-shape's-color change trials, all contributing to $\mathrm{p}$ (hits). Using the $k$ measure taken separately for no-context and shape-context trials, the age $\times$ attention interaction was significant, $F(3,100)=4.74$, $\mathrm{MSE}=0.18, \mathrm{p}<.005, \eta_{\mathrm{p}}{ }^{2}=.12$. The mean number of attended and ignored items in working memory were estimated for Grades $1-2$ at 0.57 and 0.51 ; for Grades 3-4, 0.74 and 0.67 ; for Grades 5-7, 1.08 and 0.83; and for adults, 1.46 and 1.01.

The age difference in the effects of attention, however, are most likely due at least in part to scaling factors that differ for the age groups because they are at different levels of performance. Like Cowan et al. (2011), to obtain a more valid measure of filtering, we calculated a ratio indicating the proportion of working memory items that came from the attended rather than the unattended set, calculated as $k$ (attended) $/[k$ (attended) $+k$ (unattended)]. To do this, we had to convert any negative estimates to zero. (A few children performing no better than chance in some conditions received negative capacity estimates, presumably due to random variability in the estimates, and ratios based on these estimates would be misleading.) Higher ratios indicate more tendency to filter out the lessrelevant shape. There were calculable estimates of the ratio (i.e., with non-zero denominators) for both context conditions for 23 children in the youngest group, 25 in the next group, and all 26 participants in each of the oldest two groups. Among these children, the mean ratios were $.56, .56, .57$, and $.61, \mathrm{SEM}=.03$ in each case, with no significant age effect, $F(3,96)<1, \eta_{\mathrm{p}}{ }^{2}=.02$. This finding is consistent with Cowan et al. (2011), who examined this ratio for their new data as well as for Cowan et al. (2010), and found no age effect in the ratio.

Thus, we believe that the attention effects in the less-processed scores were largely indirect effects of the age differences in the task performance levels. These performance levels could have been especially low for younger children in the present study because of the ambitious agenda of the study. To save enough time to examine context, we omitted the single-shape and $100 \%$-valid trial blocks that Cowan et al. (2010) presented before the $80 \%-20 \%$ trial block, and these initial blocks may have provided more training for children in the 2010 study. Cowan et al. (2011) did not present these initial trial blocks either, but they presented items using a much slower, serial presentation method for which less training might be needed. At any rate, the data from all three studies are consistent in suggesting that when one looks at the proportion of items in working memory coming from the more relevant shape in the $80 \%-20 \%$ condition, that proportion does not notably change with age in childhood.

\section{Discussion}

The present findings show that there are effects of the contextual support that is presented in the form of shapes indicating where colored objects were in the array to be remembered. The role of the contextual support was, however, rather limited. It only played a discernable role when the probe condition required knowledge of the object locations other than the probe object. For the new-color trials no location information was needed, only information about what set of colors occurred in the array; and for the no-change trials, what was 
sufficient was knowledge of only the color of the array item in the same location as the probe. For these conditions, context did not have a discernable effect.

When location information was needed to answer correctly, there was a benefit of context but the age groups differed in when this benefit was observed (Figure 2). The developmental trend was reduced by context for location-change probe trials, inasmuch as only the younger two age groups benefitted. In contrast, the developmental trend was increased by context in the other-shape's-color probe trials, inasmuch as only the adults benefitted. Below, we explore the use of contextual information and the conclusions that can be drawn from this use about the nature of working memory development; then we consider more broadly what we believe we know at this point about why working memory improves with age.

\section{Use of Contextual Information}

A key difference between the location-change and other-shape's-color probe trial types is that they require different kinds of inferences based on working memory, and thus afford different uses of contextual information when it is provided. In the location-change probe trials, one needs to search the working memory representation to find a match to the probe. The contextual cues could help the participant know that when a color match to the probe is found in working memory, the color went with an item of the appropriate shape at a particular location, and can be selected as the response. It can help to narrow down the location involved. This function goes well with the proposition that with development, the spatial precision of working memory representations improves (Burnett Heyes, Zokaei, van der Staaij, Bays, \& Husain, 2012; Schutte \& Spencer, 2009). Indeed, for location-change probe trials, the error analysis suggests that young children may have imprecise spatial representations of the objects and are able to benefit from context because it narrows down the spatial location of the correct item (see Table 1).

It also may happen that the probe color is not in working memory and the participant might guess that a non-remembered item was in fact that color. In the context condition, the participant could sometimes decide to select an item that was the same shape as the probe, but of an unknown color. For the location-change probe trials, the context would allow that kind of guessing to result in the correct answer half the time. Children in the two younger age groups may make that kind of guess more often than older children or adults, which also can explain why these young children not only benefit from context in the location-change condition, but also make more mistakes than the older groups in the new-color condition (by selecting instances of the probed shape instead of the door icon) .

The other-shape's-color probe trials involve potentially trickier reasoning than the locationchange probe trials. In the other-shape's-color probe trials, when a color match is found in working memory, the context cue should show that the color match to the probe was not for the appropriate shape and the probe item was therefore not in the array. However, the conflict between a match (of color) and a mismatch (of shape) may be confusing to children. A confusing situation also may arise when a color match to the probe is not found in working memory. Then the participant must consider the items that are not in working memory and decide how likely it is that an item of the same shape as the probe is also the same color. The context can help with that assessment by reminding the participant of the 
shapes of non-remembered items. Children apparently more often resolved the situation by selecting an array location, whereas adults were more able to use context to infer that the exact item was probably not in the array. In the context condition, the extra errors that children made were mostly responses to an array location with the correct shape, but incorrect color in the array (see error analyses).

It may seem surprising that the children in Grades 5 through 7, in late childhood through early adolescence, performed as well as adults in the other-shape's-color probe trials with no context (Figure 2), yet did not benefit from context as adults did. This difference could occur because adults are better able to think about contextual cues that could help lead to the inference that the probe item was not in the array. Adults may resolve the conflict better if they detect a color match to the probe (where there is not a shape match), and they may use context better if they do not detect a color match to the probe. Although areas of the brain responsible for retaining items in working memory may approach adult-like function by these late childhood years, the brain by no means reaches adult-like status by that age, and continues to improve in ways that may be needed to make the more complex inferences using working memory: frontal and parietal structure and function (Casey et al., 2000; Scherf, Sweeney, \& Luna, 2006; Thomason, Race, Burrows, Whitfield-Gabrieli, Glover, \& Gabrieli, 2009), areas important for attention-based processing such as the anterior cingulate (Scherf et al., 2006), maturation of association cortex (Gogtay et al., 2004), and white matter density (Sowell, Peterson, Thompson, Welcome, Henkenius, \& Toga, 2003).

Given that the contextual support was equally useful when the probe was in the moreattended or the less-attended shape, it appears that participants did not try to combine some items into a configuration that only included the more-attended items, but were able to consider all items together. Performance was still better for the more-often-tested shape, so this overall representation did include a greater resource allocation to the more-often-tested shape.

Context to remind participants of the spatial configuration of the array in working memory might have been expected to play a role in all probe conditions. One might have further expected that younger children would benefit more from this contextual support in all conditions, given that the development of the ability to form mental configurations is an important aspect of spatial long-term memory development (Clements-Stephens, McKellJeffers, Maddux, \& Shelton, 2011; Lehnung et al., 1998; Newcombe, Uttal, \& Sauter, 2013; Uttal, 1996). The reason context cues do not play a more ubiquitous role in our study, we suspect, is that a configuration can be formed of the four array items that often contains enough information to bind items' shapes to their locations (e.g., Jiang et al., 2000; Treisman \& Zhang, 2006; Woodman et al., 2003). Given that there were only two shapes, shape information could be assigned to spatial locations in a memory configuration, using grouping, more easily than could colors. In principle, one would only have to remember the placement of an imaginary line segment ending in two circles, and another line segment ending in two triangles. This configural information would make the contextual cues unnecessary, provided that the representations were sufficiently precise in spatial location. It could be for this reason that contextual reminders provided a benefit only in a few 
conditions (for young children when spatial precision is needed, and for adults when a complex inference sometimes must be applied).

\section{Why Does Working Memory Improve with Childhood Development?}

We still do not understand the basis of the increase in working memory performance with age in childhood. It could be the result of an increase in the number of slots available to store items (e.g., an expanding focus of attention or the ability to activate more schemes with maturation as in some neo-Piagetian view) or it could be the result of faster refreshment of activated long-term memory by an attention-demanding process, keeping more schemes active before they can decay (as in the time-based resource sharing model, e.g., Camos \& Barrouillet, 2011; Gaillard, Barrouillet, Jarrold, Camos, 2011). What the present evidence adds is that spatial precision may play a role, and the use of sophisticated inferences when a match is not found may make a difference for performance.

Our strategy in recent research has been to seek situations in which an alternative factor is controlled, to see whether an age difference in working memory is still found. Cowan, Ricker, Clark, Hinrichs, and Glass (in press) found that the developmental increase from 6 years to adulthood in the ability to remember items from an array, viewed in normalized scores, was practically unattenuated when the stimuli in the array were unfamiliar characters as opposed to familiar letters. We also found an age increase in working memory using simple sentences for which the different age groups displayed equal knowledge (Gilchrist, Cowan, \& Naveh-Benjamin, 2009). In research on other confounding processes using visual arrays, we found a developmental increase even when age groups were equivalent in the ability to filter out less-relevant stimuli and focus attention on the more relevant stimuli (Cowan et al., 2010). The same was found when array items were presented slowly, one at a time, with or without covert verbal rehearsal impeded (Cowan, AuBuchon, Gilchrist, Ricker, $\&$ Saults, 2011). Thus, evidence for the basic capacity hypothesis appears to be building and the present work adds to the story by showing that memory for the configuration of items is a somewhat minor aspect of working memory responsible for the developmental increase.

According to the neo-Piagetian school of thought, there is a developmental increase in the number of items that can be kept active at once using attention (e.g., Alp, 1994; Burtis, 1982; Case, 1995; Morra, 2000; Pascual-Leone \& Johnson, 2011). Although this hypothesis is attractive for its simplicity and intuitive appeal, it is difficult to validate. Throughout the years, investigators have pointed out different alternative factors that could explain working memory development. For example, Chi (1978) implicated knowledge by showing that working memory for chess configurations was better in children with good chess knowledge than for adults with much less chess knowledge. Similarly, Case, Kurland, and Goldberg (1982) implicated knowledge in a finding that adults' memory for lists was reduced to the level ordinarily found in 6-year-olds when the adults' stimuli were lists of numbers in a foreign language, bringing down adults' speed of identifying the spoken stimuli to match the speed at which 6-year-olds identified numbers in their native language. The present findings add to research results showing the limited contribution of various factors, converging on the notion that there is a developmental increase in storage capacity. 
Another possible developmental difference that could contribute to the findings is that the ability to bind multiple feature of an object may improve with age (Brockmole \& Logie, 2013; Cottini et al., 2014; Cowan, Naveh-Benjamin, Kilb, \& Saults, 2006). Although Luck and Vogel (1997) suggested that encoding an object into working memory results in all features being entered into working memory, recent work has shown that memory for features trades off. In adults, the requirement to maintain both shape and color results in fewer colors being remembered than when only color has to be remembered, and fewer shapes being remembered than when only shape has to be remembered (Cowan, Blume, \& Saults, 2013; cf. Hardman \& Cowan, in press; Oberauer \& Eichenberger, 2013). Four colors with their shapes and locations are likely to be way over young children's capabilities, let alone adults. It will take additional work, however, to pin down the exact role of feature binding in the development of working memory.

In sum, we have shown that there is only a limited benefit of providing a rich context to support recognition of a probe item in a working memory representation of an array of objects (colored shapes) and that the nature of this benefit changes from the early elementary school years to adulthood. Although there are various developmental factors that are likely to contribute to working memory development (various types of knowledge, strategies, and attention mechanisms), the field still awaits a method that could directly assess the role of possible increases in basic working memory slots or resources.

\section{Acknowledgments}

We thank Bret Glass and Garrett Hinrichs for assistance. This research was conducted with support from NIH Grant R01-HD21338. Address correspondence to Nelson Cowan, Dept. of Psychological Sciences, McAlester Hall, University of Missouri, Columbia, MO 65211.

\section{References}

Alp IE. Measuring the size of working memory in very young children: The imitation sorting task. International Journal of Behavioral Development. 1994; 17:125-141.

Brady TF, Tenenbaum JB. A probabilistic model of visual working memory: Incorporating higher order regularities into working memory capacity estimates. Psychological Review. 2013; 120:85109. [PubMed: 23230888]

Brockmole JR, Logie RH. Age-related change in visual working memory: a study of 55,753 participants aged 8-75. Frontiers in Psychology. 2013; 4:1-5. Article 12. [PubMed: 23382719]

Burnett Heyes S, Zokaei N, van der Staaij I, Bays PM, Husain M. Development of visual working memory precision in childhood. Developmental Science. 2012; 15:528-539. [PubMed: 22709402]

Burtis PJ. Capacity increase and chunking in the development of short-term memory. Journal of Experimental Child Psychology. 1982; 34:387-413. [PubMed: 7153702]

Camos V, Barrouillet P. Developmental change in working memory strategies: From passive maintenance to active refreshing. Developmental Psychology. 2011; 47:898-904. [PubMed: 21417518]

Case, R. Capacity-based explanations of working memory growth: A brief history and reevaluation.. In: Weinert, FE.; Schneider, W., editors. Memory performance and competencies: Issues in growth and development. Erlbaum; Mahwah, NJ: 1995. p. 23-44.

Case R, Kurland DM, Goldberg J. Operational efficiency and the growth of short-term memory span. Journal of Experimental Child Psychology. 1982; 33:386-404.

Casey BJ, Giedd JN, Thomas KM. Structural and functional brain development and its relation to cognitive development. Biological Psychology. 2000; 54:241-257. [PubMed: 11035225] 
Chen Z, Cowan N. Working memory inefficiency: Minimal information is utilized in visual recognition tasks. Journal of Experimental Psychology: Learning, Memory, \& Cognition. 2013; 39:1449-1462.

Chi, MTH. Knowledge structures and memory development.. In: Siegler, R., editor. Children's thinking: What develops?. Erlbaum; Hillsdale, NJ: 1978.

Chong SC, Treisman A. Representation of statistical properties. Vision Research. 2003; 43:393-404. [PubMed: 12535996]

Chong SC, Treisman A. Statistical processing: computing the average size in perceptual groups. Vision Research. 2005; 45:891-900. [PubMed: 15644229]

Clements-Stephens AM, McKell-Jeffers GO, Maddux J, Shelton AL. Strategies for spatial organization in adults and children. Visual Cognition. 2011; 19:886-909.

Cottini M, Pieroni L, Spataro P, Devescovi A, Longobardi E, Rossi-Arnaud C. Feature binding and the processing of global-local shapes in bilingual and monolingual children. Memory \& Cognition. Epub ahead of print. 2014 DOI 10.3758/s13421-014-0467-1.

Cowan N. The magical number 4 in short-term memory: A reconsideration of mental storage capacity. Behavioral and Brain Sciences. 2001; 24:87-185. [PubMed: 11515286]

Cowan N. Working memory underpins cognitive development, learning, and education. Educational Psychology Review. 2014; 26:197-223. [PubMed: 25346585]

Cowan N, AuBuchon AM, Gilchrist AL, Ricker TJ, Saults JS. Age differences in visual working memory capacity: Not based on encoding limitations. Developmental Science. 2011; 14:10661074. [PubMed: 21884322]

Cowan N, Blume CL, Saults JS. Attention to attributes and objects in working memory. Journal of Experimental Psychology: Learning, Memory, and Cognition. 2013; 39:731-747.

Cowan N, Elliott EM, Saults JS, Morey CC, Mattox S, Hismjatullina A, Conway ARA. On the capacity of attention: Its estimation and its role in working memory and cognitive aptitudes. Cognitive Psychology. 2005; 51:42-100. [PubMed: 16039935]

Cowan N, Fristoe NM, Elliott EM, Brunner RP, Saults JS. Scope of attention, control of attention, and intelligence in children and adults. Memory \& Cognition. 2006; 34:1754-1768. [PubMed: 17489300]

Cowan N, Morey CC, AuBuchon AM, Zwilling CE, Gilchrist AL. Seven-year-olds allocate attention like adults unless working memory is overloaded. Developmental Science. 2010; 13:120-133. [PubMed: 20121868]

Cowan N, Naveh-Benjamin M, Kilb A, Saults JS. Life-Span development of visual working memory: When is feature binding difficult? Developmental Psychology. 2006; 42:1089-1102. [PubMed: 17087544]

Cowan N, Ricker TJ, Clark KM, Hinrichs GA, Glass BA. Knowledge cannot explain the developmental growth of working memory capacity. Developmental Science. in press.

Cowan N, Saults JS, Blume CL. Central and peripheral components of working memory storage. Journal of Experimental Psychology: General. 2014; 143:1806-1836. [PubMed: 24867488]

Gaillard V, Barrouillet P, Jarrold C, Camos V. Developmental differences in working memory: Where do they come from? Journal of Experimental Child Psychology. 2011; 110:469-479. [PubMed: 21664622]

Gathercole SE, Pickering SJ, Ambridge B, Wearing H. The structure of working memory from 4 to 15 years of age. Developmental Psychology. 2004; 40:177-190. [PubMed: 14979759]

Gilchrist AL, Cowan N, Naveh-Benjamin M. Investigating the childhood development of working memory using sentences: New evidence for the growth of chunk capacity. Journal of Experimental Child Psychology. 2009; 104:252-265. [PubMed: 19539305]

Gogtay N, Giedd JN, Lusk L, Hayashi KM, Greenstein D, Vaituzis AC, et al. Dynamic mapping of human cortical development during childhood through early adulthood. PNAS. 2004; 101:81748179. [PubMed: 15148381]

Halford GS, Cowan N, Andrews G. Separating cognitive capacity from knowledge: A new hypothesis. Trends in Cognitive Sciences. 2007; 11:236-242. [PubMed: 17475538] 
Hardman K, Cowan N. Remembering complex objects in visual working memory: Do capacity limits restrict objects or features? Journal of Experimental Psychology: Learning, Memory, and Cognition. in press.

Jiang YV, Capistrano CG, Palm BE. Spatial working memory in children with high-functioning autism: Intact configural processing but impaired capacity. Journal of Abnormal Psychology. 2014; 123:248-257. [PubMed: 24661175]

Jiang Y, Olson IR, Chun MM. Organization of visual short-term memory. Journal of Experimental Psychology: Learning, Memory, and Cognition. 2000; 26:683-702.

Lehnung M, Leplow B, Friege L, Herzog A, Ferstl R, Mehdorn M. Development of spatial memory and spatial orientation in preschoolers and primary school children. British Journal of Psychology. 1998; 89:463-480. [PubMed: 9734301]

Luck SJ, Vogel EK. The capacity of visual working memory for features and conjunctions. Nature. 1997; 390:279-281. [PubMed: 9384378]

Miller GA. The magical number seven, plus or minus two: Some limits on our capacity for processing information. Psychological Review. 1956; 63:81-97. [PubMed: 13310704]

Newcombe, NS.; Uttal, DH.; Sauter, M. Spatial development.. In: Zelazo, PD., editor. The Oxford handbook of developmental psychology (Vol 1): Body and mind. Oxford University Press; New York, NY, US: 2013. p. 564-590.

Rouder JN, Morey RD, Morey CC, Cowan N. How to measure working-memory capacity in the change-detection paradigm. Psychonomic Bulletin \& Review. 2011; 18:324-330. [PubMed: 21331668]

Sowell ER, Peterson BS, Thompson PM, Welcome SE, Henkenius AL, Toga AW. Mapping cortical change across the human life span. Nature Neuroscience. 2003; 6:309-315.

Woodman GF, Vecera SP, Luck SJ. Perceptual organization influences visual working memory. Psychonomic Bulletin \& Review. 2003; 10:80-87. [PubMed: 12747493]

Morra S. A new model of verbal short-term memory. Journal of Experimental Child Psychology. 2000; 75:191-227. [PubMed: 10666325]

Oberauer K, Eichenberger S. Visual working memory declines when more features must be remembered for each object. Memory \& Cognition. 2013; 41:1212-1227. [PubMed: 23716004]

Pascual-Leone, J.; Johnson, J. A developmental theory of mental attention: Its applications to measurement and task analysis.. In: Barrouillet, P.; Gaillard, V., editors. Cognitive development and working Memory: From neoPiagetian to cognitive approaches. Psychology Press; Hove, UK: 2011. p. 13-46.

Riggs KJ, McTaggart J, Simpson A, Freeman RPJ. Changes in the capacity of visual working memory in 5 to 10yearolds. Journal of Experimental Child Psychology. 2006; 95:18-26. [PubMed: 16678845]

Scherf KS, Sweeney JA, Luna B. Brain basis of developmental change in visuospatial working memory. Journal of Cognitive Neuroscience. 2006; 18:1045-1058. [PubMed: 16839280]

Schutte AR, Spencer JP. Tests of the dynamic field theory and the spatial precision hypothesis: Capturing a qualitative developmental transition in spatial working memory. Journal of Experimental Psychology: Human Perception and Performance. 2009; 35:1698-1725. [PubMed: 19968430]

Shore DI, Burack JA, Miller D, Joseph S, Enns JT. The development of change detection. Developmental Science. 2006; 9:490-497. [PubMed: 16911451]

Thomason ME, Race E, Burrows B, Whitfield-Gabrieli S, Glover GH, Gabrieli JE. Development of spatial and verbal working memory capacity in the human brain. Journal of Cognitive Neuroscience. 2009; 21:316-332. [PubMed: 18510448]

Treisman A, Zhang W. Location and binding in visual working memory. Memory \& Cognition. 2006; 34:1704 1719. [PubMed: 17489296]

Uttal DH. Angles and distances: Children's and adults' reconstruction and scaling of spatial configurations. Child Development. 1996; 67:2763-2779. [PubMed: 9071762]

Wheeler ME, Treisman AM. Binding in short-term visual memory. Journal of Experimental Psychology: General. 2002; 131:48-64. [PubMed: 11900102] 
Xu Y, Chun MM. Visual grouping in human parietal cortex. PNAS. 2007; 104:18766-18771. [PubMed: 17998539] 


\section{Highlights}

- Visual working memory capacity increases during childhood, but why?

- One possible factor is the use of the spatial configuration of items.

- We tested memory for arrays of colored shapes with and without configuration cues.

- Children in early elementary school years were helped by configuration cues at test.

- Configuration did not, however, account for all of the development of capacity. 


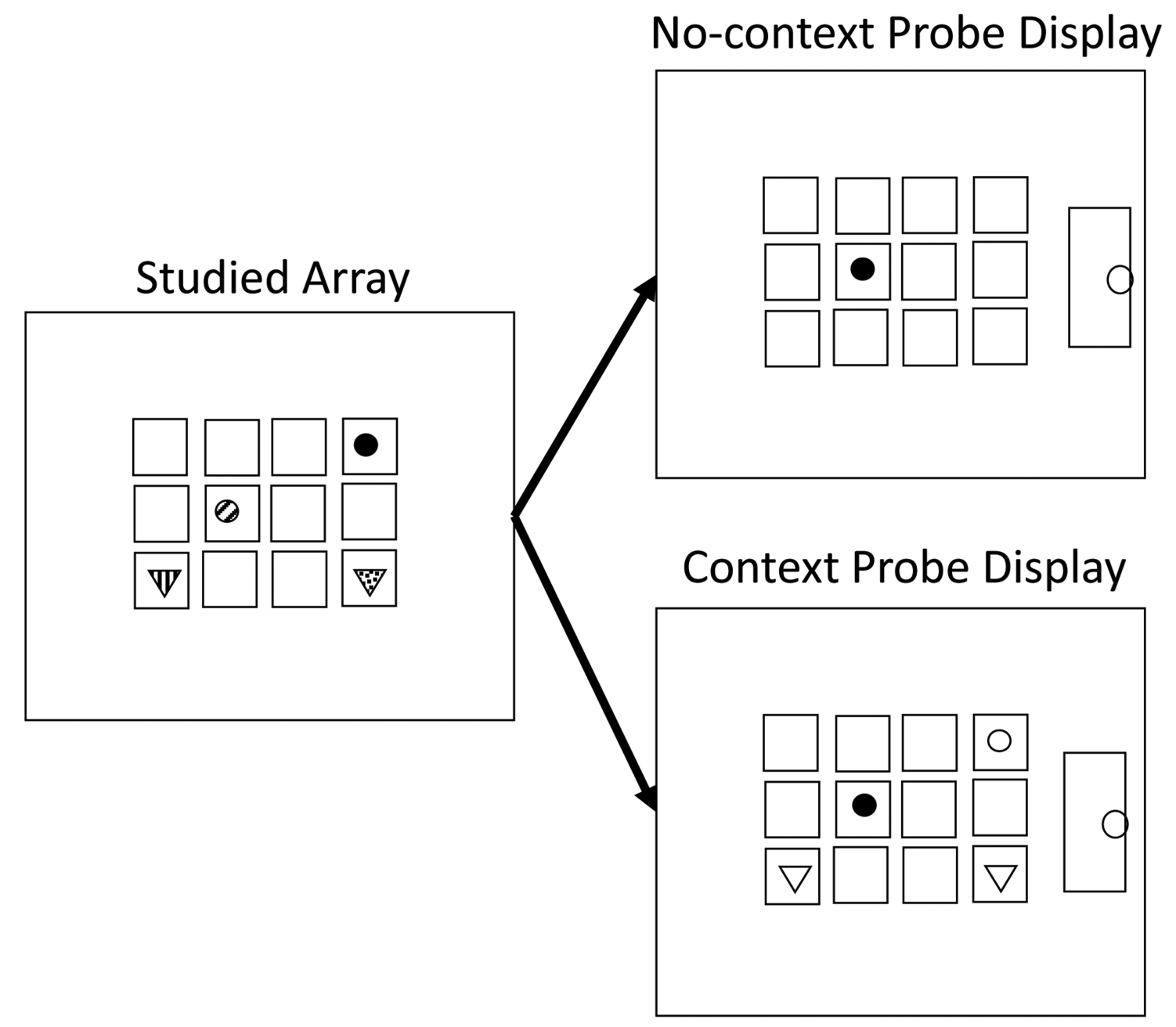

Figure 1.

Illustration of a studied array (left) followed by a probe display (response screen) on a trial with no context present (top right) or with contextual cues present (bottom right). Striped, dotted, and solid fill patterns represent colors in the experiment. The empty shapes in the shape-context probe correctly indicate where a colored item of that same shape was found in the studied array. The task was to indicate where the filled circle of the same color was found in the array; if nowhere, the correct response was to click on the door. The probe object always appeared where an object of that same shape had been, though the probe color could be any color in the stimulus set depending on the condition. In the trial shown, the correct response was a mouse click on the upper, right location of the grid. 

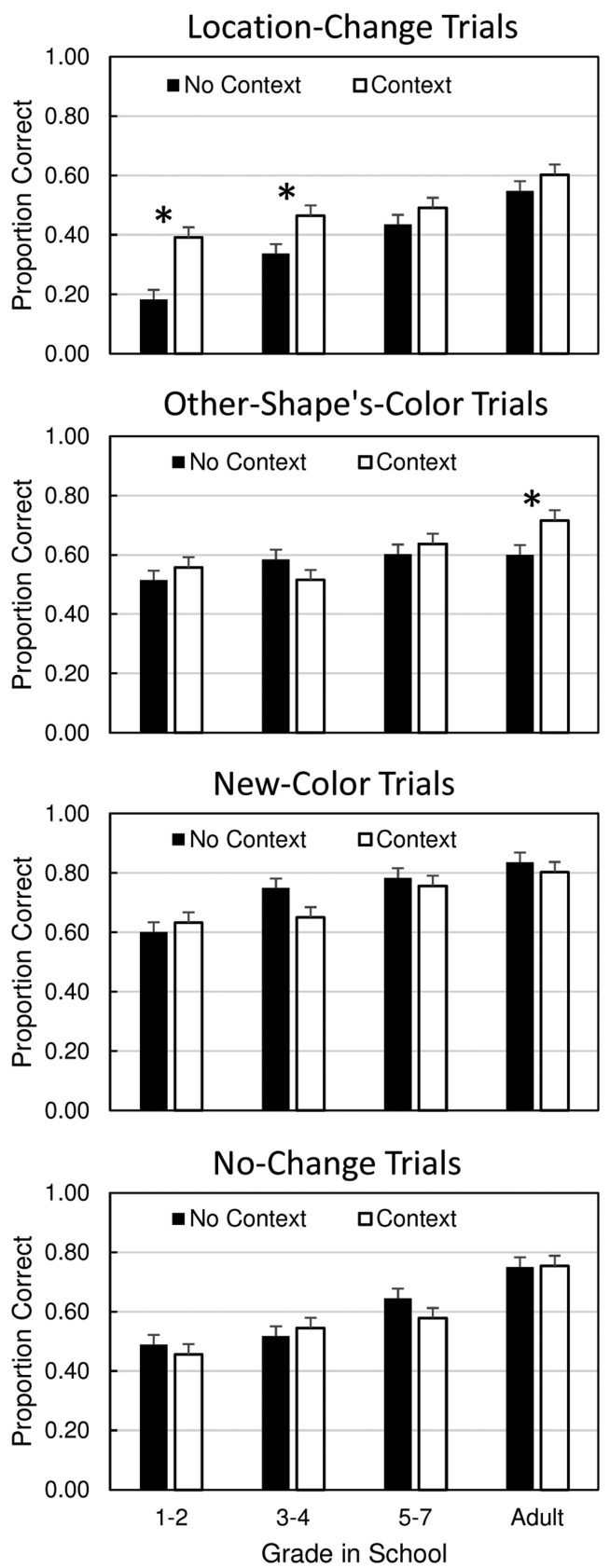

Figure 2.

For each probe condition (four separate panels as labeled), the no-context (dark bars) and context (light bars) proportion correct responding in each age group (x axis). Error bars are standard errors. Significant post-hoc tests showing an effect of context for a particular age group with a particular trial type are shown with an asterisk $(*)$; there were only three such instances. 


\section{Table 1}

Proportion of responses for each error type in the no-context and context versions of location-change probe trials, in groups that benefitted from context

\begin{tabular}{lcccc}
\hline & \multicolumn{2}{c}{ No Context } & \multicolumn{2}{c}{ Context } \\
Erroneous Response & Mean & SE & Mean & SE \\
\hline \multirow{4}{c}{ Grades 1-2 in School } \\
"No change" & 0.25 & 0.03 & 0.19 & 0.03 \\
"Not in array" & 0.39 & 0.03 & 0.37 & 0.04 \\
Wrong other location & 0.18 & 0.03 & 0.05 & 0.02 \\
& \multicolumn{5}{c}{ Grades 3-4 in School } \\
"No change" & 0.19 & 0.03 & 0.19 & 0.03 \\
"Not in array" & 0.35 & 0.03 & 0.30 & 0.04 \\
Wrong other location & 0.12 & 0.03 & 0.04 & 0.02 \\
\hline
\end{tabular}

J Exp Child Psychol. Author manuscript; available in PMC 2016 July 01. 